\title{
OS PRINCÍPIOS DE ADAPTAÇÃO E OPTIMIZAÇÃO NO PROCESSO DE ESTRUTURAÇÃO DAS ORGANIZAÇÕES.
}

\begin{abstract}
O dinamismo e a imprevisibilidade do ambiente põem em destaque o problema de adaptação da empresa às alterações deste. Na resolução do problema torna-se extremamente importante o tipo de estrutura organizacional, uma vez que somente as estruturas organizacionais flexíveis são capazes de assegurar uma rápida adaptação da organização às alterações ambientais. A flexibilidade da estrutura depende tanto da diversificação e do tipo de produção como do método de estruturação utilizado na elaboração da estrutura.

Neste artigo dedicamos a nossa atenção ao problema da estruturação das organizações num contexto de optimização e apresentamos o método de optimização "step-by-step", baseado no uso dos algoritmos de "cluster analysis". Este método é orientado na optimização e avaliação da estrutura nos vários níveis de hierarquia ao longo da elaboração da estrutura. A abordagem de optimização "stepby-step" permite iniciar o processo de reestruturação a partir de um qualquer nivel de hierarquia, o que possibilita uma rápida análise das capacidades de adaptação da organização às alterações das condições de funcionamento.
\end{abstract}

Valeria Reva*

Palavras-chave: estrutura organizacional, métodos de optimização, processos de adaptação.

The dynamism and unpredictability of the environment raises a problem of adaptation of the company to the changes involved. The fast adaptation of the organization to changes can only be assured by flexible organizational structures, which is why the type of structure becomes extremely important. The flexibility of the structure depends on the diversification and type of production as well as on the method of structural synthesis.

\footnotetext{
* Instituto Universitário de Desenvolvimento e Promoção Social - Pólo de Viseu do Centro Regional das Beiras da Universidade Católica Portuguesa.
} 
In this paper we consider the problem of organizational structuring from the standpoint of optimization, and present the method of "stepby-step" optimization, based on the use of cluster analysis algorithms. This method permits the structure to be optimized and estimate at the different levels of hierarchy during the process of structuring, and the process of reorganization to be initiated from any level of hierarchy. This makes it possible to quickly analyse the capabilities of adaptation of the organization to changes in functioning conditions.

Key words: organizational structure, methods of optimization, adaptation processes.

\section{INTRODUÇÃO}

A vida de uma empresa, qualquer que seja a sua natureza, torna-se cada vez mais complicada devido aos inúmeros obstáculos exteriores e interiores que influenciam o seu funcionamento e devem ser ultrapassados para que esta consiga atingir os objectivos propostos.

A concorrência de mercado estimula o desenvolvimento das novas tecnologias de produção, capazes de baixar os custos de produção e os níveis dos preços de mercado. O progresso técnico acelera-se e as empresas devem, sem demora, seguir a evolução. Nesta fase, o principal problema em destaque é a possibilidade de adaptar o sistema de produção já existente às novas tecnologias com as despesas mínimas de remodelação. No entanto, a partir de uma certa altura, torna-se insuficiente competir só pelo uso das tecnologias mais modernas, pois estas são já uma realidade adquirida e, assim sendo, as empresas dedicam-se com particular atenção à elaboração de estratégias de marketing alternativas. Torna-se importante a capacidade que a empresa revela em analisar o mercado, prever o futuro dos produtos, conhecer o comportamento das empresas concorrentes, analisar as consequências tanto das suas acções como das acções dos potenciais concorrentes, ou seja, de uma fase do progresso meramente técnico passamos a uma fase do progresso técnico-informativo. A expectativa na obtenção de lucros futuros e aquisição de um certo grau de poder no mercado é o motivo pelo qual as empresas investem os próprios recursos na pesquisa de novas tecnolologias de produção e novos produtos. Entretanto, o mercado continua a viver absorvendo tudo quanto de novo aparece e exigindo esforços cada vez mais significativos que permitam fazer face às suas leis de concorrência livre.

Como tal, existe uma necessidade de adaptação da empresa (vamos designá-la como organização) à complexidade, dinamismo e imprevisibilidade do meio em que se insere. Este problema pode ser resolvido através da flexibilidade da estratégia global da organização. No entanto, a simples boa vontade dos gestores em fazer face à concorrência, 
não é suficiente enquanto base para o desenvolvimento de uma estratégia nova. Na formação de uma estratégia, um dos pontos de partida são, necessariamente, os activos reais existentes e a actividade operacional da organização, ou seja, a estrutura organizacional existente que permite, ou não, à organização seguir a nova estratégia. Assim sendo, para existir a flexibilidade da estratégia é preciso criar as estruturas organizacionais flexíveis que sejam capazes de assegurar uma rápida adaptação da organização às alterações ambientais.

Por outro lado, uma boa estruturação é um factor da maior importância para se alcançar êxito na gestão, uma vez que é impossível gerir bem uma organização mal estruturada. A estruturação deficiente pode passar despercebida caso a empresa beneficie de uma boa situação financeira, ou se estiver num mercado privilegiado: à medida que os problemas surgem, os mesmos podem ser resolvidos sem grandes alterações de fundo. Os efeitos de uma má estruturação só são visíveis e prejudicam a longo prazo.

Tudo isto levanta uma série de questões sobre o problema da estruturação e eficácia das organizações: a abordagem destas questões constitui o propósito deste artigo.

\section{O ESSENCIAL DA ESTRUTURA ORGANIZACIONAL}

Antes de definir o próprio conceito de estrutura organizacional vamos referir as investigações em que se obtiveram os resultados mais significativos no desenvolvimento do problema da estruturação eficaz das organizações.

Exigem uma especial atenção os estudos empíricos seguintes (MINTZBERG, 1995; FAURES, 1991):

- O estudo realizado por Joan Woodward (1965) que aborda a relação entre a estrutura e o sistema técnico de produção;

- Os estudos realizados por Burns e Stalker (1961), Paul Lawrence e Jay Lorsch (1967), Galbraith (1977), que descreveram o impacto do ambiente na estrutura organizacional;

- O estudo de Derek Pug (1963 - 1964) que analisa a correspondência entre dimensões da organização e características da estrutura.

Estes estudos, apesar de considerarem aspectos diferentes do funcionamento das organizações, todos chegaram à mesma conclusão geral de que não existe uma estrutura que seja melhor, mas sim diferentes estruturas que melhor se adaptam a diferentes condições.

Mas a descoberta mais importante foi o resultado dos estudos de Pradip Khandwalla (1971-1974) e Child (1977) que analisam as co-variações entre os parâmetros da estrutura nas empresas de desempenho elevado e de 
desempenho medíocre: o desempenho não se encontra significativamente correlacionado com nenhuma variável estrutural considerada isoladamente, mas provém de uma combinação das variáveis de estrutura.

A mesma conclusão apresentou no seu relatório (1973) o grupo sueco dos Institutos Escandinavos para a Investigação Administrativa (SIAR): “... a fonte principal da ineficácia e dos conflitos é a falta de coerência entre os subsistemas da organização" (MINTZBERG, 1995, p.249).

Numerosos estudos sobre a eficácia estrutural, feitos nos anos 80, deram origem a uma variedade de interpretações do conceito "estrutura organizacional". Alguns autores acentuavam a distribuição das tarefas entre os elementos da estrutura organizacional, considerando a formação da estrutura como distribuição formal ou informal das tarefas entre indivíduos ou meios técnicos. Outros autores consideravam a estrutura organizacional como um conjunto de relacionamentos de um determinado tipo, existentes em organizações, concentrando-se no tratamento da organização como um sistema de fluxos de natureza diferente. Assim, apesar de terem um avanço significativo no desenvolvimento da teoria das organizações, a maioria dos estudos abordava este assunto sem a tentativa de fazer uma síntese que permitisse agrupar todas as descobertas. Como resultado, "a maior parte da literatura ... não relaciona a descrição da estrutura de uma organização com o seu funcionamento" (MINTZBERG, 1995, p.30).

A união destas abordagens diferentes associada a uma visão completa das relações e interacções entre os diversos factores que influenciam a estrutura organizacional, relacionando-os com a coerência resultante entre os elementos dessa estrutura organizacional, permite a abordagem de sistema ao conceito da estrutura organizacional. As hipóteses de base desta abordagem são as seguintes:

\section{1) Uma organização é um sistema.}

O conceito de sistema que vamos referir, é o conceito fundamental da teoria dos sistemas de informação. Se considerarmos que os últimos têm a finalidade de optimizar a comunicação e o processo de decisão nas organizações e que se desenvolvem a partir da estrutura organizacional já existente e, de certa forma, reflectem esta estrutura organizacional, a definição do sistema utilizado deve coincidir com a noção da organização.

$\mathrm{Na}$ teoria dos sistemas de informação (RASCÃO, 2001) o sistema é definido como um conjunto de componentes inter-relacionados que actuam em simultaneidade de forma a atingir objectivos comuns, aceitando dados de entrada (inputs) e produzindo resultados (outputs) numa organizada consequência de processos de transformação. Como funções básicas do sistema consideram-se: input - recolha dos elementos que entram no sistema para serem processados; tratamento - processo de transformação 
que converte os elementos de entrada em produto acabado; output resultado do processo de transformação, ou seja, o produto acabado; armazenamento - armazenamento temporário dos elementos.

$\mathrm{Na}$ teoria das organizações, uma organização (um sistema) é um conjunto de elementos de dimensões e natureza diferentes. Os elementos são inter-relacionados e actuam juntos para atingir o principal objectivo da organização - maximização do seu valor. Os elementos de entrada para a organização são os elementos necessários para a produção (ou para os efeitos dos serviços exercidos) e os elementos necessários para a gestão (p.ex. a informação). $\mathrm{O}$ tratamento envolve os processos tecnológicos de produção, o acto de serviço, o processamento das decisões ou informação, etc. A função de armazenamento tanto pode ser ligada à produção como à informação. Os elementos de saída são os produtos, serviços, decisões, informação, etc..

2) Uma organização é um sistema complexo: os componentes têm origem e características diferentes; os relacionamentos entre os componentes, representados pelos fluxos, têm natureza diferente (fluxos materiais, de informação, financeiros, etc.).

3) Uma organização é um sistema complexo aberto, o que significa que os componentes do sistema directa ou indirectamente são afectados pela influência do meio ambiente.

$\mathrm{O}$ acima referido justifica a seguinte definição do sistema, que também assumimos como a definição da organização:

Definição 1. Sob o sistema $\boldsymbol{S}$ considere-se um conjunto de elementos $\boldsymbol{S}=(\boldsymbol{M}, \boldsymbol{R}, \boldsymbol{N}, \boldsymbol{X}, \boldsymbol{Y}, \boldsymbol{O}, \boldsymbol{F})$, onde $\boldsymbol{M}$ é o conjunto dos elementos do sistema (instrumentos e objectos de trabalho, recursos humanos, tarefas de gestão, informação, etc.), que têm natureza diferente; $\boldsymbol{R}$ é o conjunto das relações entre os elementos do conjunto $\boldsymbol{M} ; \boldsymbol{N}$ é o conjunto dos elementos do ambiente, que entram em interacção com os elementos do conjunto $\boldsymbol{M} ; \boldsymbol{X}$ é o conjunto de inputs do sistema (as matérias primas, os produtos semi-fabricados, a energia, o esforço humano, a informação, etc.); $\boldsymbol{Y}$ é o conjunto de outputs do sistema (os produtos acabados, serviços, a informação, etc.); $\boldsymbol{O}$ é o conjunto de relações entre os elementos do conjunto $\boldsymbol{M U N} ; \boldsymbol{F}$ é o conjunto das funções, que definem as dependências entre as entradas e saidas.

Desta forma, a estrutura organizacional será definida pelo conjunto dos elementos, características deles, relações entre eles e relações entre os elementos do sistema e o ambiente.

Um sistema pode ser constituído por vários sub-sistemas, que são sistemas de dimensão inferior em relação ao sistema inicial e realizam 
tarefas especializadas relacionadas com os objectivos globais do sistema inicial.

A separação nos sub-sistemas tem como finalidade a diminuição das dificuldades de análise dos sistemas complexos. Os sub-sistemas, normalmente, são separados a partir de determinadas características que são comuns para os elementos, por exemplo, homogeneidade das entradas e saídas, o tipo das relações, o grupo das funções, etc. A análise do sistema complexo, baseada no estudo dos sub-sistemas e correlações entre eles, diminui a quantidade dos elementos e relações a considerar simultaneamente mas, ao mesmo tempo, permite abranger todas as características do sistema total.

Consideramos uma organização o conjunto formado por um sub-sistema de produção (objecto de gestão) e um sub-sistema de gestão, correlacionados entre si. No seguimento deste trabalho vamos designá-las simplesmente por sistema de produção e sistema de gestão. Por conseguinte, a estrutura organizacional será definida como o conjunto da estrutura do sistema de produção, estrutura do sistema de gestão, correlações entre eles e correlações entre eles e o ambiente.

\section{AS ABORDAGENS DO PROBLEMA DE ESTRUTURAÇÃO. O PROBLEMA DA OPTIMIZAÇÃO DA ESTRUTURA ORGANIZACIONAL}

A estrutura organizacional, que condicione os mecanismos de coordenação (a maneira como e em que níveis da gestão as decisões são tomadas, como se efectua a transferência das directivas entre os elementos do sistema e o controlo do desempenho das directivas), é o principal responsável pela eficácia da gestão e funcionamento da organização. Por esta razão, no problema de estruturação da organização, tem-se notado a tendência para uma elaboração da forma estrutural óptima da organização.

Distinguem-se duas principais abordagens do problema de elaboração do projecto da estrutura organizacional (ZVIRKUN, 1982).

A primeira abordagem é mais orientada para a elaboração do projecto do sistema de gestão da organização, como base da estrutura organizacional. Nesta abordagem a forma estrutural óptima seria aquela que permite a gestão eficaz, sendo as despesas ligadas à gestão, mínimas. Nesta área podemos separar os grupos de estudo seguintes:

- $\mathrm{O} 1^{\mathrm{o}}$ grupo de estudos dedicava-se ao processo de formação de uma rede de objectivos e tarefas, que se considera como o modelo hierárquico dos objectivos e tarefas correlacionadas do sistema total. $\mathrm{O}$ ponto de partida era o pressuposto de possibilidade de estabelecimento de um objectivo global da organização. Considere-se que este objectivo global 
pode ser conseguido por meio da realização de uma consequência dos subobjectivos e sub-tarefas, chegando assim a uma hierarquia de objectivos e tarefas. No caso mais vulgar, uma estrutura organizacional elabora-se de seguinte modo: a uma entidade ou sub-divisão estrutural distribui-se uma só tarefa ou um só objectivo, assegurando assim uma correspondência unívoca entre modelo hierárquico dos objectivos e estrutura organizacional. Nesta estrutura cada "superior" tem como subordinados apenas elementos estruturais de níveis mais baixos, responsáveis pela realização das tarefas necessárias para conseguir o objectivo estipulado pelo "superior". É óbvio que, nesta situação, um "subordinado" devia ter apenas um único "superior", o que, eventualmente, vai levar à duplicação do mesmo trabalho e utilização irracional dos recursos disponíveis. Com o aumento da dimensão da organização estas estruturas tornam-se ainda mais complexas e, completamente, inflexíveis, o que reduz, significativamente, a possibilidade de adaptação da organização às alterações das condições de funcionamento.

- O $2^{\circ}$ grupo de estudos, tendo estabelecido o objectivo global, apresentou-o de uma forma analítica. Definido em seguida o conjunto das limitações para o funcionamento do sistema, este grupo de estudos considerava o processo de funcionamento da organização como o processo de desenvolvimento do problema da optimização. Devido à sua complexidade, este problema da optimização não poderia ser facilmente resolvido sem ser decomposto em vários sub-problemas. Assim, o processo de desenvolvimento da estrutura organizacional consistia, simplesmente, na decomposição baseada num critério de qualidade, do problema inicial em sub-problemas. As sub-divisões do sistema de gestão da organização foram encarregadas de resolver os sub-problemas.

- $\mathrm{O} 3^{\circ}$ grupo de estudos dedicava-se à elaboração da função que reflectia a qualidade de funcionamento do sistema de gestão. Como argumentos desta função consideravam os parâmetros da estrutura organizacional assumidos como mais importantes e a própria função foi apresentada em forma de soma ponderada destes parâmetros. Depois da elaboração do sistema das limitações, baseadas nas condições de funcionamento da organização e exigências apresentadas à organização, resolviam o problema da optimização. Assim sendo, elaboraram a estrutura organizacional óptima conforme a resolução do problema da optimização.

- $\mathrm{O} 4^{\circ}$ grupo de estudos elaborava a estrutura organizacional por meio de uma divisão hierárquica do conjunto dos elementos do sistema em sub-conjuntos, baseada na estimativa quantitativa dos relacionamentos entre os elementos do sistema. Neste caso, como aspectos principais que determinavam a estrutura organizacional, foram escolhidos os fluxos existentes dentro da organização (principalmente, fluxos materiais e de informação). 
A segunda abordagem está relacionada com a elaboração do projecto do sistema de produção a partir da tecnologia de produção e, em seguida, do projecto de sistema de gestão. A ideia que proclamavam os estudos deste ramo era a seguinte: o objectivo e a razão da existência do sistema de gestão não é a gestão das operações em forma de tomada de decisões, a partir da troca e elaboração da informação, mas sim a gestão da distribuição dos recursos materiais. Por isso, considera-se a estrutura dos fluxos materiais como determinante da estrutura organizacional, base para o sistema da informação. Assim, primeiro constrói-se a estrutura óptima dos fluxos materiais, e depois elabora-se a estrutura hierárquica do sistema de gestão, designando a estrutura óptima da organização.

Analisando os estudos feitos no domínio da resolução do problema de elaboração e optimização da estrutura organizacional, podemos constatar o seguinte:

- Os estudos são caracterizados pelo desenvolvimento unilateral do problema da elaboração e optimização da estrutura organizacional: a análise desenvolve-se ou do ponto de vista dos objectivos da organização ou do ponto de vista dos recursos existentes;

- Sob a estrutura da organização, principalmente, entende-se a estrutura do sistema de gestão, relacionando o problema da optimização da estrutura da organização simplesmente com a elaboração da estrutura óptima do sistema de gestão;

- Muitos métodos funcionam mais como esquemas conceptuais, sendo extremamente complicados para a aplicação em organizações reais;

- Alguns estudos foram orientados na utilização dos esquemas típicos existentes de distribuição dos recursos ou tomada de decisões, transferindo, assim, formas organizacionais típicas não efectivas dentro do próprio modelo;

- Alguns estudos dedicaram-se principalmente à análise dos mecanismos de coordenação do trabalho e consideravam-nos como elementos fundamentais da estrutura.

$\mathrm{Na}$ realidade, a principal causa dos defeitos dos modelos analíticos de elaboração da estrutura óptima encontra-se na complexidade das organizações. Torna-se, simplesmente, impossível, num só modelo analítico, exprimir todos os relacionamentos existentes dentro da organização e entre a organização e o ambiente. A abordagem de sistema na elaboração do projecto da estrutura organizacional permite resolver este problema. A divisão do sistema em sub-sistemas e, em seguida, a diminuição da complexidade permite a elaboração do modelo global de estruturação da organização sob a forma de um conjunto dos modelos mais simples, relacionados entre si através dos fluxos de entradas e saídas. 
Contudo isso ainda não é a real vantagem da abordagem de sistema. Imaginemos que foi elaborado um projecto da estrutura organizacional considerada como óptima. As conclusões sobre a capacidade desta estrutura organizacional em cumprir os objectivos da organização só podem ser tiradas depois da organização começar a funcionar. Verificar a qualidade do projecto, desta forma, será dispendioso. Por isso, torna-se muito importante estimar a eficácia do projecto antes que este seja introduzido numa organização real. Considerando a organização como um sistema é possível estimar o projecto a partir dos resultados de imitação do funcionamento da organização com a estrutura elaborada: os modelos de estruturação organizacional funcionam como base para o desenvolvimento do sistema de imitação de funcionamento (CHUNG, (2004).

\section{A ESTRUTURA ORGANIZACIONAL E O AMBIENTE}

De facto, para compreender como as organizações se estruturam, temos que entender como é que funcionam e qual é a finalidade da actividade da organização. Os objectivos e a estratégia da organização surgem como resultado da interacção da organização com o ambiente.

A organização faz parte do ambiente e, tal como o ambiente influencia o funcionamento da organização, a organização influi sobre o ambiente (Figura 1). O ambiente, numa forma mais global, pode ser considerado como o conjunto de três sistemas caracterizados pelas suas próprias estruturas: sistema político, sistema económico e sistema sócio-cultural. Estes sistemas influenciam a organização directa ou indirectamente e definem "as regras do jogo" para todos os elementos do ambiente. No caso da economia planificada, onde o estado se apresenta como "o monopolista da vida das empresas", as directivas destes três sistemas acabam por ser os principais parâmetros a ter em conta ao longo do desenvolvimento e funcionamento da organização. No caso da economia de livre concorrência faz sentido considerar isoladamente dois elementos do ambiente que se encontram em contacto quotidiano, mais visível e influente, isto é, as organizações-clientes e as organizações-concorrentes. Estes dois elementos vão introduzir parâmetros adicionais e são, na verdade, os principais responsáveis pela existência do dimamismo do ambiente.

Figura 1

A ESTRUTURA ORGANIZACIONAL NO ÂMBITO DE FUNCIONAMENTO 


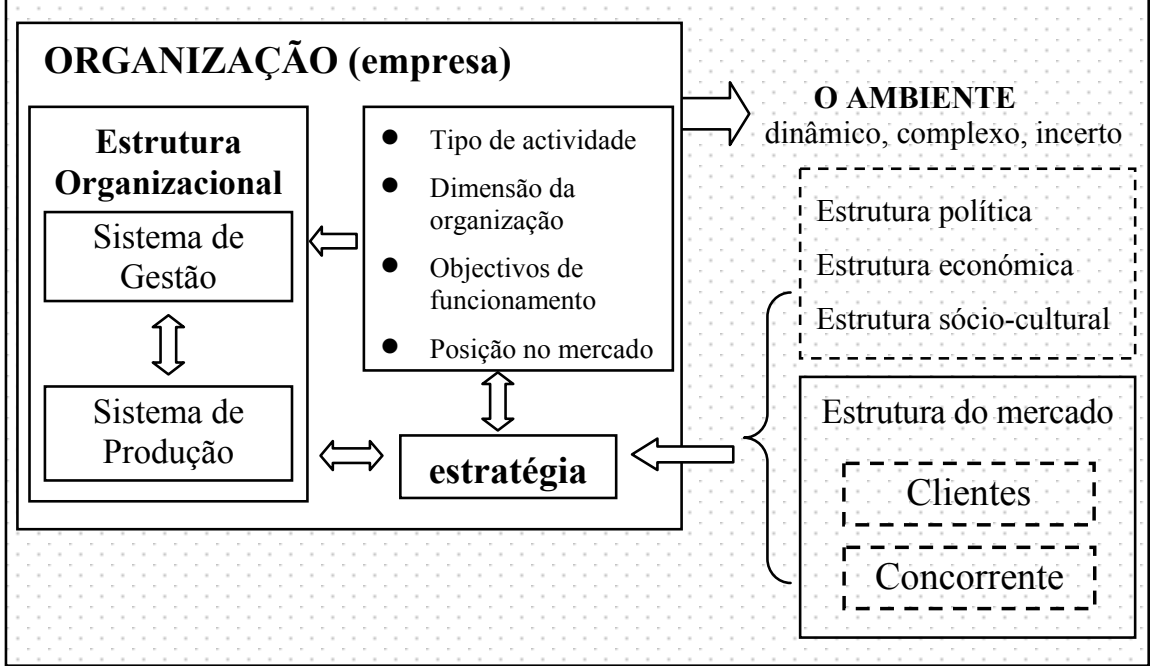

Fonte: Elaborado pela autora

\section{A ESTRUTURA ORGANIZACIONAL E OS CUSTOS}

Há duas razões para que os economistas se preocupem com os custos (HAY, 1993). A primeira tem a sua origem na eficiência do alocamento de recursos do ponto de vista do interesse social. O conceito de custo, neste caso, deve entender-se como o custo de oportunidade: o uso do recurso na produção de um bem impossibilita o seu uso na produção de um bem alternativo. O custo dos recursos para a sociedade é o valor destes no melhor uso alternativo. A segunda razão concentra-se na firma mais do que no alocamento de recursos na economia global. A ideia é que os custos, em certa medida, determinam os preços; os preços, por sua vez, determinam a parcela do mercado; tudo isto, em conjunto, determina a rentabilidade da empresa.

A importância dos custos para o funcionamento da organização é indiscutível. A teoria dos custos examina vários tipos de custos e vários aspectos da influência dos custos na eficácia do funcionamento da organização. Isto, por sua vez, origina vários modelos de estruturação organizacional, baseados no princípio de minimização dos custos.

Não vamos aqui apresentar as expressões analíticas desenvolvidas ao longo da tentativa de apreciar determinados custos, mas iremos analisar os tipos de custos a suportar pela organização do ponto de vista da 
possibilidade a estimá-los e formalizá-los para os efeitos de elaboração do projecto da estrutura organizacional.

Numa forma mais ampla, os custos totais podem ser classificados em directos e indirectos (MENEZES, 1988). Os custos directos, na sua maioria, têm natureza quantitativa e envolvem os explícitos e implícitos. Os custos explícitos abrangem as despesas (financeiras, administrativas e de produção) previamente estabelecidas. Os custos implícitos compreendem os custos de oportunidade resultantes da não realização das estratégias alternativas. Os custos indirectos são principalmente de natureza qualitativa e relacionam-se com o aumento dos custos directos futuros por razões de alteração da imagem da empresa no mercado (p.ex, aumento do risco financeiro da empresa).

Esta classificação dos custos chama a nossa atenção para a natureza quantitativa e qualitativa dos mesmos e para o momento de aparecimento dos custos - custos actuais ou custos futuros.

A classificação de custos também pode ser abordada em duas perspectivas diferentes: a económica e a financeira.

Na perspectiva económica, os custos são separados em dois grupos (SANTOS, 1997):

custos variáveis, que são directamente proporcionais às quantidades produzidas ou vendidas, e encargos da estrutura, que não são directamente proporcionais às quantidades produzidas, ou seja, custos fixos e custos variáveis não proporcionais.

Na perspectiva financeira, a actividade económica exige a aplicação dos recursos financeiros, determinando assim, o custo do capital utilizado no investimento em activos fixos ou circulantes, apresentado analiticamente como soma ponderada do custo de capital próprio e capital alheio.

Precisamente, os capitais próprios devem ser remunerados conforme os desejos dos titulares do capital social da empresa, dando origem ao conceito de custo dos capitais próprios. Os capitais próprios devem ser valorizados, podendo o oposto dar origem aos custos implícitos. Os empréstimos a curto, a médio e longo prazo devem ser remunerados a uma taxa de juro. Este custo, que faz parte do custo dos capitais alheios, também varia dependendo do tipo da taxa de juro (nominal ou efectiva) e da forma de pagamento dos juros (antecipados ou postecipados) e pode ser ainda aumentado devido às comissões, impostos ou despesas administrativas. O crédito obtido da parte dos fornecedores implica a perda de eventuais descontos de pronto pagamento. $\mathrm{O}$ uso exagerado deste tipo de crédito pode piorar as condições de aprovisionamento ou até causar problemas neste. $\mathrm{O}$ crédito concedido aos clientes significa uma privação dos recursos financeiros que poderiam ser aplicados e remunerados, dando origem aos custos de oportunidade. A existência de stocks também exige 
uma certa mobilização dos capitais próprios ou alheios e origina determinados custos.

Justifica-se uma elevada atenção dos economistas para o problema dos custos, dado que estes são responsáveis pelos valores de uma variedade de indicadores, que são relevantes para a análise da viabilidade da organização (Figura 2).

Figura 2

A ESTRUTURA ORGANIZACIONAL E OS CUSTOS

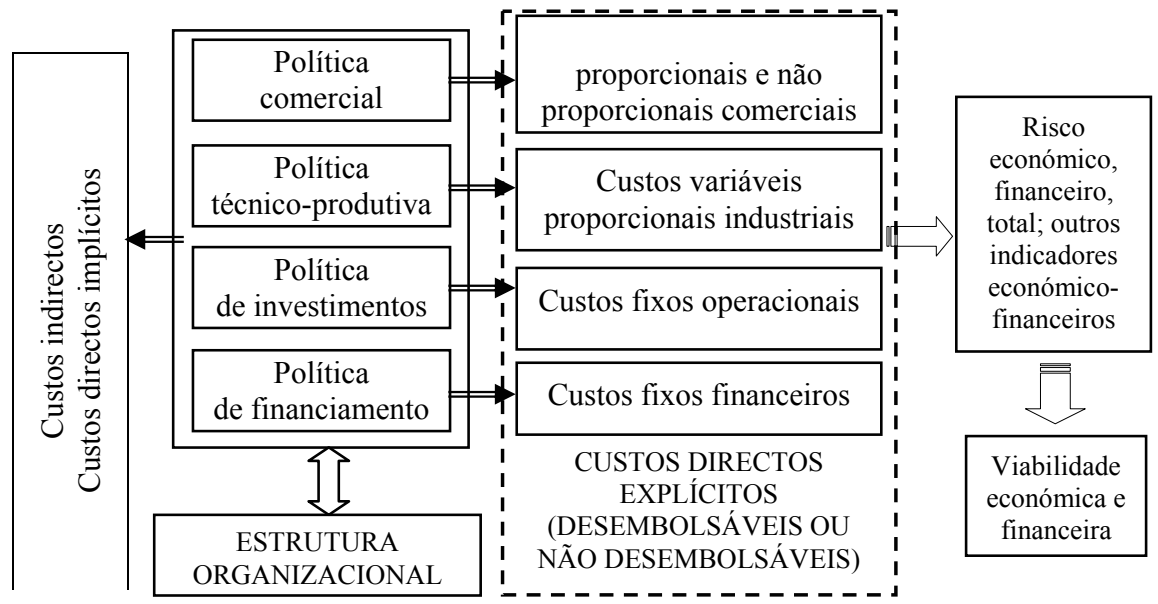

Fonte: Elaborado pela autora

Para dar um exemplo simples vamos considerar dois conceitos que assumem uma grande importância na Gestão Financeira, precisamente, os conceitos de risco económico e financeiro, e a relação destes últimos com os custos a suportar pela organização. Os indicadores considerados como principais na análise do risco económico e financeiro são Ponto Morto Económico (PME), Grau de Alavanca Operacional (GAO), Ponto Morto Financeiro (PMF) e Grau de Alavanca Financeira (GAF) (PEYRARD, 1992; MENEZES, 1988).

"O efeito económico de alavanca resulta unicamente da existência de custos fixos e permite-nos analisar a capacidade da empresa para utilizar uma certa e determinada estrutura técnico-produtiva, comercial e administrativa no sentido de maximizar os resultados de exploração; ..." (MENEZES, 1988: p.58). Quando uma empresa tem um peso elevado de custos fixos, ela atinge o seu limite de rentabilidade (PME) com um volume elevado de produção, o que implica um risco económico elevado. Logo que o limite de rentabilidade é atingido, começa a funcionar o efeito 
económico de alavanca e um ligeiro aumento/diminuição das vendas provoca um aumento/diminuição significativo do resultado operacional. Pelo contrário, quando a empresa tem um baixo peso dos custos fixos, o limite de rentabilidade pode ser atingido com um baixo nível de vendas, mas um pequeno aumento de vendas provoca um ligeiro aumento do resultado operacional. Assim, o PME e GAO, como medida do efeito económico de alavanca, constituem os instrumentos relevantes para a análise preliminar da viabilidade económica de investimentos em activo fixo (política dos investimentos), que contribui para a existência dos custos fixos. Apesar disso, o conceito de PME também chama a nossa atenção para os parâmetros, ligados à política técnico-produtiva e comercial que são o preço de venda unitário e o custo de venda unitário.

O risco económico e a estrutura de capital utilizada (peso dos capitais próprios e capitais alheios no capital total) são determinantes do risco financeiro. A existência de encargos financeiros pode ter um efeito positivo ou negativo sobre a rentabilidade dos capitais próprios, o que se analisa através da formulação analítica do efeito financeiro de alavanca. $\mathrm{O}$ conceito da PNF permite avaliar o risco financeiro na perspectiva da tesouraria, chamando a atenção para a existência de custos fixos que no fundo não são desembolsáveis (amortizações, reintegrações e provisões) (MENEZES, 1988: p.73).

Esta breve análise visualiza que:

- o problema da minimização dos custos pode ser abordado em várias perspectivas, dependendo da importância dada a um certo tipo de custos. Por exemplo, nas duas abordagens da elaboração de uma estrutura organizacional óptima (parágrafo 3 do artigo) de várias formas foram mencionados os custos a suportar pela empresa: na primeira abordagem a optimização foi orientada na minimização dos custos de gestão; na segunda abordagem a estrutura óptima dos fluxos materiais percebia praticamente a minimização dos custos de produção.

- uma certa combinação de custos pode influenciar de maneira diferente a rentabilidade operacional e financeira da organização e, em seguida, a viabilidade económica e financeira da mesma organização. Por isso, ocorrem muitas dificuldades no desenvolvimento de um único modelo analítico, baseado na teoria da minimização de custos, que permita uma avaliação completa da estrutura organizacional.

\section{UM MÉTODO DE OPTIMIZAÇÃO DA ESTRUTURA ORGANIZACIONAL}

Ao longo do desenvolvimento do método foram seguidos, como principais, os seguintes objectivos: 
$1^{\mathrm{o}}$ : atribuir ao sistema de informação a propriedade de proporcionar a informação referente, não somente à performance passada e presente da organização, mas também à performance futura resultante das alterações estruturais possíveis.

$2^{\circ}$ : em vez de resolver o problema de estruturação a partir de um critério global de optimização (como, p. ex. minimização de custos), resolvê-lo como um problema multi-criterial, onde a optimização dos critérios se efectua ao longo da elaboração do projecto da estrutura, ou seja, num nível de hierarquia pode ser optimizado um critério.

$3^{\text {o: }}$ desenvolver um método que permita uma análise rápida das possibilidades de adaptação da organização às novas condições de funcionamento.

Neste caso, para diminuir a complexidade do problema de estruturação, decompusemos a organização em dois sub-sistemas - sub-sistema de produção e sub-sistema de gestão. Como os sub-sistemas são sistemas de dimensão inferior, justifica-se a aplicação da definição do sistema anteriormente referida aos sub-sistemas de produção e gestão. Entretanto, é preciso ter um certo cuidado na aplicação da definição, desde que os elementos $\boldsymbol{M}, \boldsymbol{R}, \boldsymbol{N}, \boldsymbol{X}, \boldsymbol{Y}, \boldsymbol{O}, \boldsymbol{F}$ dos sub-sistemas tenham as suas próprias especificações.

Vamos definir o sistema de produção (o objecto de gestão) como $S_{1}=\left(M_{1}, R_{1}, N_{1}, X_{1}, Y_{1}, O_{1}, F_{1}\right)$ onde:

○ $\quad \boldsymbol{M}_{1}$ é o conjunto dos elementos, separados do conjunto $\boldsymbol{M}$ pelo princípio de participação directa no processo da produção. Neste conjunto são incluídos os diferentes tipos de equipamento, processos tecnológicos e recursos humanos;

○ $\boldsymbol{R}_{\boldsymbol{I}}$ é o conjunto das relações entre os elementos do conjunto $\boldsymbol{M}_{\boldsymbol{1}}$;

○ $\quad \boldsymbol{N}_{\boldsymbol{l}}$ é o conjunto dos elementos do ambiente, que entram em interacção com os elementos do conjunto $\boldsymbol{M}_{\boldsymbol{I}}$. Enquanto elementos do ambiente consideram-se o sub-sistema de gestão, os fornecedores das matérias-primas e os consumidores dos produtos da organização;

○ $\boldsymbol{X}_{1}$ é o conjunto de inputs do sistema, os quais são: as matérias primas, os produtos semi-fabricados, a energia, as decisões tomadas no sistema de gestão;

- $\quad \boldsymbol{Y}_{1}$ é o conjunto de outputs do sistema, os quais são: os produtos acabados ou semi-acabados e a informação sobre o estado dos elementos $M_{1}$;

○ Os conjuntos $\boldsymbol{O}_{\boldsymbol{1}}$ e $\boldsymbol{F}_{\boldsymbol{1}}$ são definidos pelo processo tecnológico e pelo tipo das relações entre os elementos do conjunto $M_{I} U N_{I}$;

O sistema de gestão define-se como $\boldsymbol{S}_{2}=\left(\boldsymbol{M}_{2}, \boldsymbol{R}_{2}, \boldsymbol{N}_{2}, \boldsymbol{X}_{2}, \boldsymbol{Y}_{2}, \boldsymbol{O}_{2}, \boldsymbol{F}_{2}\right)$, onde:

$\circ$ Os elementos do conjunto $\boldsymbol{M}_{\boldsymbol{2}}$ são grupos de pessoal; aglomerados de informação diferentes; meios de recolha, agrupamento, 
tratamento e armazenamento da informação; métodos de processamento das decisões, etc.;

○ $\boldsymbol{R}_{2}$ é o conjunto das relações entre os elementos do conjunto $\boldsymbol{M}_{2}$;

- $\boldsymbol{N}_{2}$ é o conjunto dos elementos do ambiente, os quais são: o objecto de gestão, as organizações fornecedoras e os consumidores, outras organizações do ambiente que têm relações com a organização em consideração;

○ $\quad \boldsymbol{X}_{2}$ e $\boldsymbol{Y}_{2}$ são aglomerados diferentes de informação;

$\circ \boldsymbol{O}_{2}$ apresenta toda a variedade de inter-relações entre os elementos do sub-sistema;

$\circ \boldsymbol{F}_{2}$ é o conjunto das funções, relacionadas com as formas de tratamento de informação.

Os conjuntos $\boldsymbol{M}_{1}$ e $\boldsymbol{M}_{2}$ são os conjuntos dos elementos-base das estruturas do sistema de produção $\boldsymbol{S}_{1}$ e sistema de gestão $\boldsymbol{S}_{2}$.

$\mathrm{Na}$ literatura dedicada à elaboração do projecto da estrutura organizacional do sistema complexo (estudos sobre as estruturas das grandes empresas industriais), considera-se como estrutura organizacional óptima o resultado do compromisso entre exigências da tecnologia de produção e simplicidade de gestão do sistema total.

O sistema de gestão mais simples é aquele que tem só uma unidade de gestão. Entretanto, para um sistema complexo, composto por uma variedade numerosa de elementos correlacionados, é impossível criar um sistema de gestão, que tenha um só elemento e consiga uma gestão completa, oportuna e eficaz. Assim sendo, a estrutura do sistema de gestão deve ser a estrutura hierárquica, pois só assim é permitido reduzir a complexidade da própria tarefa de gestão de um objecto complexo.

Uma vez que a elaboração do projecto da estrutura organizacional se baseia na coordenação do objecto de gestão e sistema de gestão, seria racional elaborar o projecto do sistema de gestão a partir do projecto do sistema de produção, elaborado anteriormente. Esta abordagem da estruturação da organização considera a optimização step-by-step da estrutura organizacional (SCURIKHIN, V.I., ZABRODSKY, V.A., 1992).

O aspecto principal da elaboração do projecto da estrutura do objecto de gestão $\boldsymbol{S}_{1}$ (ou sistema de gestão $\boldsymbol{S}_{2}$ ) é a resolução do problema de agrupamento em hierarquia dos elementos-base do sistema $\boldsymbol{S}_{\boldsymbol{1}}$ (sistema $\boldsymbol{S}_{2}$ ). Normalmente, quando se trata de elaboração da estrutura organizacional de um sistema complexo, assume-se que o conjunto dos elementos-base dos sistemas de gestão e produção são definidos e dados a priori. Esta abordagem não é aceitável para as organizações que pretendem, com uma certa regularidade, renovar a nomenclatura dos produtos produzidos (ou serviços) ou, por razões de remodelação do produto anteriormente produzido, renovar a tecnologia de produção. As alterações da nomenclatura ou alterações no processo tecnológico, podem alterar o 
conjunto das operações a exercer pelos elementos-base da estrutura ou até criar a necessidade de reformulação dos próprios elementos-base da estrutura. Por isso, o processo de elaboração do projecto de qualquer um dos sistemas será iniciado pela resolução do problema de síntese do conjunto dos elementos-base.

Assim, a primeira etapa da optimização step-by-step da estrutura organizacional consiste na resolução do problema da síntese dos elementos-base da estrutura do objecto de gestão $\boldsymbol{S}_{\boldsymbol{I}}$ - síntese do conjunto $M_{1}$.

A segunda etapa consiste na elaboração do projecto do sistema $\boldsymbol{S}_{\boldsymbol{1}}$ a partir do agrupamento dos elementos do conjunto $\boldsymbol{M}_{\boldsymbol{1}}$ em hierarquia com uso dos algoritmos de hierarchical cluster analysis. Como resultado, o sistema $\boldsymbol{S}_{\boldsymbol{I}}$ pode ser apresentado como um conjunto de sub-sistemas: $S_{1}=\left\{S_{i j}^{1}\right\}$, onde $\boldsymbol{i}$ determina o nível de hierarquia, e $\boldsymbol{j}$ determina o número do sub-sistema para o nível de hierarquia $\boldsymbol{i}$. Os elementos-base do conjunto $\boldsymbol{M}_{1}$ são agrupados em conjuntos $M_{i j}^{1}$ seguindo a regra $M_{i j}^{1} \supset\left\{M_{i-1 j}^{1}\right\}$, ou seja, o conjunto dos elementos-base no nível de hierarquia $\boldsymbol{i}$ engloba os conjuntos dos elementos-base do nível de hierarquia $\boldsymbol{i}-\mathbf{1}$.

A estrutura do objecto de gestão $\boldsymbol{S}_{\boldsymbol{1}}$ elabora-se tendo em conta, por um lado, certos objectivos e, por outro, limitações. As limitações na sua maioria são diferentes, dependendo dos níveis de hierarquia e da especificação da produção. Entretanto, existem as limitações comuns, tais como a homogeneidade dos elementos que formam os grupos de cada nível de hierarquia, as normas administrativas, as limitações para o nível de relacionamento entre os elementos e entre os grupos de elementos.

A homogeneidade dos elementos assume um carácter diferente de um nível de hierarquia para o outro. Por exemplo, na formação de um nível preocupamo-nos com a homogeneidade dos processos tecnológicos a realizar, na formação de outro nível preocupamo-nos com a homogeneidade do equipamento utilizado ou dos processos de montagem, etc.

As normas administrativas predeterminam o número dos elementos subordinados, o que permite a gestão eficaz destes. Empiricamente, foi estabelecido que ao passar-se dos baixos níveis aos níveis de hierarquia mais elevados as normas administrativas aumentam 2-4 vezes. Os limites para as normas administrativas variam dependendo da experiência do gestor, automatização dos processos de produção e de gestão.

Entre os elementos do sistema de produção existem fluxos materiais e de informação. Os volumes destes fluxos são diferentes para as várias estruturas organizacionais, mas nenhum ultrapassa os limites que determinam a eficácia de utilização destes fluxos. 
Em geral, o problema da síntese de estrutura do sistema $\boldsymbol{S}_{\boldsymbol{I}}$ pode ser formulado do seguinte modo: agrupar os elementos-base do conjunto $\boldsymbol{M}_{\boldsymbol{l}}$, de forma a elaborar a estrutura hierárquica com os elementos a satisfazer as limitações preestabelecidas e os critérios de optimização para os elementos, a chegar aos seus valores extremos.

Os elementos executivos, que compõem o objecto de gestão, efectuam as suas funções só no caso de existência dos elementos de gestão, que realizam a função de gestão. Isto significa que dentro de um elemento do objecto de gestão podem ser realizadas tanto funções de produção como funções de gestão dos processos de produção. Por isso, a estrutura hierárquica do sistema $\boldsymbol{S}_{1}$ vai definir os elementos-base do sistema de gestão (o conjunto $\boldsymbol{M}_{2}$ ). A síntese do conjunto $\boldsymbol{M}_{2}$ será a terceira etapa da optimização step-by-step. A quarta etapa consiste na elaboração da própria estrutura do sistema de gestão $\boldsymbol{S}_{2}$, resultante da elaboração coerente das estruturas linear, funcional e administrativa do sistema de gestão.

A finalidade da abordagem de estruturação e optimização step-by-step pretende, tendo em consideração as limitações estruturais e funcionais, elaborar a estrutura organizacional $\boldsymbol{S}^{*}$, que possibilita a maior eficiência do funcionamento da organização.

Ou seja, $S^{*}=\underset{S \in \mathbf{S}}{\arg \max _{l}} \mu_{l}\left(\phi_{l}(f(S))\right)$, onde $\mathbf{S}$ é o conjunto das $S \in \mathbf{S}$

possíveis variantes da estrutura organizacional que satisfazem as limitações estruturais e funcionais; $f(S)$ - o conjunto das funções locais para estimar a eficácia da estrutura; $\phi_{l}, \mu_{l}$ - os operadores que permitem passar de um conjunto de estimativas locais de funcionamento para uma estimativa generalizada de eficácia de estrutura.

Vamos considerar mais pormenorizadamente os problemas a resolver ao longo da optimização step-by-step.

O início do desenvolvimento do projecto de estrutura do sistema de gestão, ou do sistema de produção, consiste na determinação dos conjuntos de elementos-base. Surge uma questão muito importante: o que considerar como elemento-base de um ou de outro sistema?

Como elementos-base para o objecto de gestão vamos considerar os postos de trabalho, definidos como um conjunto qualitativamente e quantitativamente organizado de meios de produção, recursos humanos, objectos de trabalho (peças ou partes constitutivas do artigo), necessários para a realização de uma determinada função de produção.

Como elementos-base para o sistema de gestão vamos considerar as unidades de gestão, definidas como um conjunto qualitativamente e quantitativamente organizado dos recursos humanos, meios e objectos de trabalho necessários para a realização de uma determinada função de gestão. 
A síntese do posto de trabalho é um problema muito complexo, que depende de vários factores inter-relacionados e, por isso, resolve-se como o problema da optimização multicriterial. O problema da síntese de postos de trabalho pode ser formalizado do mesmo modo que o problema de síntese de estrutura óptima. Precisamente, o conjunto óptimo de postos de trabalho $\boldsymbol{x}^{*}$ será definido pela equação:

$$
x^{*}=\underset{x \in \mathrm{X}}{\operatorname{argmax}} \mu_{L}\left(\phi_{L}(f(x))\right)
$$

onde $\boldsymbol{X}$ é o conjunto das possíveis variantes dos conjuntos de elementosbase que satisfazem as limitações predefinidas; $f(x)$ - o conjunto das funções locais para estimar a eficácia do posto de trabalho; $\phi_{L}, \mu_{L}-$ os operadores que permitem passar de um conjunto de estimativas locais para uma estimativa generalizada do posto de trabalho. O conjunto $X$ não é dado e deve ser formado.

A estimativa de postos de trabalho efectua-se a partir dos indicadores, que reflectem as características estruturais, técnico-organizacionais e económicas. Normalmente, como indicadores utilizam-se:

- o nível de especialização do posto de trabalho;

- o nível médio de especialização;

- o nível de automatização e mecanização;

- o coeficiente de carregamento do posto de trabalho;

- o coeficiente de carregamento do equipamento que depende do tipo de trabalho;

- $\quad$ o custo de produção para o posto de trabalho, etc.

O conjunto óptimo dos postos de trabalho define-se pela resolução do problema de agrupamento dos objectos de trabalho e operações tecnológicas, porque os outros cálculos são directos.

O esquema geral da síntese dos postos de trabalho é o seguinte:

1) $\mathrm{O}$ agrupamento dos objectos de trabalho pela equivalência. Consideram as peças ou partes constitutivas do artigo a produzir e calculam quantas vezes estas peças se encontram neste e nos vários artigos a produzir. Depois analisam o número de elementos iguais e laboriousness dos elementos para os efeitos de criação da produção especializada. Se não existir nenhum grupo destes, passam para o agrupamento pela similaridade.

2) O agrupamento dos objectos de trabalho pela similaridade.

Os grupos, resultantes do agrupamento pela equivalência, depois da análise do volume dos trabalhos necessários para produção dos elementos dos grupos, tal como as peças ou partes constitutívas soltas, entram na segunda fase do agrupamento. 
Este tipo de agrupamento baseia-se na análise das características tecnológico-construtivas. Dentro deste tipo de agrupamento separam-se os agrupamentos relacionados com as especificidades de produção e montagem, homogeneidade de materiais e detalhes semi-fabricados.

3) O agrupamento das operações tecnológicas.

Para cada peça dentro dos grupos, resultantes de agrupamento pela similaridade, analisam o processo tecnológico de produção e de montagem. Com base nisto, definem o conjunto das operações tecnológicas que, em seguida, agrupam dentro de cada grupo, resultante do agrupamento pela similaridade.

O modelo formal do problema de agrupamento dos objectos de trabalho pela similaridade é igual ao problema de agrupamento das operações tecnológicas, e é o seguinte:

Definimos como $X=\left\{x_{i}\right\}, i=\overline{1, N}$ o conjunto dos objectos de trabalho ou operações tecnológicas e como $\Psi=\left\{\varphi_{j}\right\}, j=\overline{1, m}$ o conjunto das características tecnológico-constructivas. É preciso definir o conjunto dos grupos $R=\left\{Q_{1}, \ldots, Q_{k}\right\}$ que satisfaz as condições seguintes:

$$
\begin{aligned}
& \bigcup_{i=1}^{K} Q_{i}=X, \quad Q_{k} \cap Q_{l}=0, \quad k \neq l ; \\
& \forall x_{k}, x_{l} \in Q_{i} \quad \mu\left(x_{k}, x_{l}\right) \geq k P, \quad i=\overline{1, K} ; \\
& K \rightarrow \text { min; }
\end{aligned}
$$

onde $\mu\left(x_{k}, x_{l}\right)$ é a função de similaridade; $P$ é o limite de similaridade, $k$ é o parâmetro de variação do agrupamento.

4) Determinação dos volumes do trabalho.

Baseia-se na análise dos grupos resultantes do agrupamento.

5) Definição de quantidade de postos de trabalho.

Baseia-se na análise dos grupos resultantes do agrupamento, dos volumes do trabalho, do tipo de produção, do nível de automatização da produção, etc. O principal objectivo na determinação do conjunto de postos de produção é a minimização dos custos de produção. O modelo formal do problema da síntese de estrutura do objecto de gestão é igual ao modelo (1)-(3). O conjunto $\boldsymbol{X}$, neste caso, é o conjunto de postos de trabalho. O conjunto $\Psi$ é composto por características tecnológicas, de construção, e organizacionais. Depois de a estrutura ser elaborada, efectua-se a análise da sua eficácia. Se os critérios de eficácia não estiverem satisfeitos, pode ser alterado o limite de similaridade ou reavaliado o conjunto das características, e o processo de agrupamento repete-se. O reagrupamento 
pode efectuar-se várias vezes até se chegar aos valores admissíveis dos critérios.

A estrutura do sistema $\boldsymbol{S}_{\boldsymbol{I}}$ determina os contornos do sistema de gestão $\boldsymbol{S}_{2}$, ou estrutura linear do sistema de gestão. Esta estrutura, que apresenta a divisão primária dos processos de gestão, em seguida será aperfeiçoada e transformada em estrutura funcional. A síntese da última inclui a resolução dos seguintes problemas para cada elemento da estrutura linear:

- definem-se os relacionamentos deste com os outros elementos, elaborando uma rede de fluxos informacionais;

- determina-se o conjunto de funções de gestão, tendo em conta as normas administratívas;

- determina-se o conjunto das tarefas a executar, suas características e objectivos.

Depois da análise dos dados obtidos, agrupando as tarefas de gestão e separando os blocos funcionais com relacionamentos informacionais mínimos, elaboram a estrutura funcional do sistema $\boldsymbol{S}_{2}$. Em seguida avaliam a complexidade, as características organizacionais, os relacionamentos entre os elementos do sistema construído e, caso estes não satisfaçam os critérios de eficácia, procedem à elaboração de uma nova estrutura funcional. Concluindo esta fase, podemos passar à elaboração das unidades de gestão dentro dos blocos funcionais. Como objectos de trabalho consideram-se as tarefas, caracterizadas pela tecnologia de execução, qualificação exigida de especialistas, a quem estes foram nomeados, automatização do processo de execução, etc. O esquema geral da síntese das unidades de gestão é similar ao esquema da síntese dos postos de trabalho: agrupamento das tarefas pelo princípio de equivalência e similaridade, agrupamento das operações tecnológicas, determinação dos volumes de trabalho e distribuição dos recursos humanos e, finalmente, a determinação do número das unidades de trabalho. $\mathrm{O}$ agrupamento em hierarquia das unidades de trabalho concluirá o processo de formação de estrutura do sistema de gestão. E tal como isso acontecia ao longo da elaboração da estrutura do objecto de gestão, cada passo de elaboração da estrutura do sistema de gestão será avaliado e repetido caso seja necessário. Esta possibilidade de optimização local, no fundo, era a origem da própria designação do modelo, como modelo de optimização step-by-step da estrutura organizacional.

\section{CONCLUSÃO}

Como nota final, devemos salientar que o problema de estruturação da organização, embora seja objecto de um número imenso de estudos, continua a manter-se actual, uma vez que ainda não existe uma 
metodologia completa da elaboração e aperfeiçoamento da estrutura organizacional. Na literatura existente sobre este tema faz-se referência, apenas, a uma lista de medidas necessárias para execução no acto de elaboração ou actualização do projecto da estrutura organizacional. Além disso, muitos estudos na área da organização das empresas abrangiam ou o domínio de produção, ou o domínio de administração, não reconhecendo a organização como uma coligação de elementos inter-relacionados de natureza diferente, como uma unidade dinâmica de gestão.

Neste artigo propõe-se uma outra visão do problema de estruturação da organização que foi o resultado da abordagem de sistema do conceito de estrutura organizacional e de maior atenção prestada à própria estrutura organizacional. A lógica de sistema, a utilização dos algoritmos de cluster analysis e o design da organização com os meios de high-level flow chart contribuiram para o desenvolvimento de um método de estruturação da organização que permite a elaboração da estrutura óptima da organização e pressupõe a possibilidade de simulação de funcionamento da organização com a finalidade de facilitar a adaptação desta às alterações de meio ambiente.

\section{BIBLIOGRAFIA}

CHUNG, Christopher A., (2004), Simulation modeling handbook: a practical approach, CRC Press LLC.

FAURES, Gilles, (1992), Estrutura, organização e eficácia da empresa. - Título original: Structure, organisation et efficacité de l'entreprise, Edições Cetop.

HAY, Donald A, (1993), Industrial economics and organization: theory and evidencel Donald A. Hay and Derek J. Morris, 2a edição, Oxford: Oxford University Press.

MINTZBERG, Henry, (1995), Estrutura e dinâmica das organizações. - Edição original: The structuring of organizations (1979), $1^{\text {a }}$ edição, Publicações Dom Quixote, Lisboa.

MENEZES, H. Caldeira, (1988), Princípios de gestão financeira, $2^{\mathrm{a}}$ edição, Editorial Presença, Lisboa.

RASCÂO, José, (2001), Sistemas de informação para as Organizações - a informação chave para a tomada de decisão, $1^{\mathrm{a}}$ edição, Edições Silabo, Lisboa.

PEYRARD, Josette, (1992), Gestão financeira com exercícios. - Título original: Gestion Financière avec exercice, $2^{\mathrm{a}}$ edição, Publicações Dom Quixote, Lisboa.

SANTOS, Arlindo Fernandes, (1997), Análise financeira: conceitos, técnicas e aplicações, $3^{\mathrm{a}}$ edição, INIEF. Economia e Gestão Lisboa.

ZVIRKUN, A. D., (1982), The basis of the synthesis of the structure of the complex systems. Moskow: Nauka. (em Russo)

SCURIKHIN, V.I., ZABRODSKY, V.A., et al., (1992), The automatization of organizational design of enterprises. Kiev: Tekhnika. (em Russo) 